\title{
Integrating occupant behaviour in the simulation of coupled electric and thermal systems in buildings
}

\author{
Ruben Baetens * Dirk Saelens \\ Building Physics Section, Department of Civil Engineering, K.U.Leuven, Belgium \\ *ruben.baetens@bwk.kuleuven.be
}

\begin{abstract}
The presented work depicting the integrated modelling of probabilistic occupant behaviour in buildings consists of non-physical modelling with a physical multidomain impact. The human behaviour considering occupancy, the use of lighting and the use of electric appliances in dwellings has been implemented, but the same method can be used for other stochastic behaviour. The stochastic behaviour is used for simulation of coupled thermal and electrical systems in the building stock and is of high importance for the assessment of smart grids and distributed energy generation. Implementing stochastic occupant behaviour influences the internal heat gains which in turn influence the heat load of the building and the switch-on and -off moment of e.g. an electric heat pump. This, together with the power demand of the used electric appliances and possible on-site generation determine the load on the electric grid and possible instabilities. Here, the use of deterministic profiles for use at both the building and the building district scale no longer fits.
\end{abstract}

Comparison between a determinsitic approach as proposed in ISO 13790 and the use stochastic profiles shows that the direct first order effect is on average rather small: the difference in total internal gains and its influence on the indoor temperature averages nearly zero and the standard deviations $\sigma$ are small, however high peaks may occur. Also the difference in effect on the electric distribution grid voltage averages nearly zero, however here strong peaks occur which are of most importance for the grid stability. When taking in account the second order effect of heating by means of electricity, much larger differences are noticed: due to longer and more differentiated occupancy times, the average indoor temperature rises. Furthermore, the moment of heating differentiates compared to a determinsitic approach resulting in more but smaller peak demands towards the electricity grid.

Keywords: Stochastic modelling; Occupant be- haviour; Grid load; Thermal building response.

\section{Introduction}

Since the development of dynamic building simulation programs such as TRNSYS and ESP-r in the mid-70's $[14,26]$, the assessment of comfort and energy demand of buildings has been subject of intensive research. As a recast of the European legislation 2002/91/EG obliges all members to build nearly zero-energy buildings by 2020, the need of detailed dynamic simulations still increases. The recast should result in the implementation of renewable energy in the building stock, most often resulting in an all-electrical solution with a combination of building-integrated photovoltaic systems (BIPVs) and an electrical heat pump where the total electricity consumption is. However, a problem of simultaneity between electricity production and consumption arises with the distribution grid as virtual storage [3, 4]. This paper focuses on the modelling of user behaviour, influencing both the fluctuations in the electricity demand as well as the thermal demand.

\section{Stochastic behaviour}

The behaviour of building occupants is most often simplified in current practice as deterministic schedules of user behavior as inputs to the building simulation model (e.g. as in ISO 13790 [12]), whereas the probabilistic user behaviour plays an important role. The combination of both stochastic and controllable local service demand, and both stochastic and controllable local energy conversion in different energy vectors (i.e. heat, cold, fuel or electricity in buildings) allows to operate [7] and optimize the energy distribution and control in many different ways at both the scale of a single building and the building district .

The modelling of human behaviour is implemented in Modelica as it is part of a bigger approach including 
thermal building simulation, simulation of thermal energy systems, electrical energy systems and distributed generation (see Fig.1).

The presented work consists of the non-physical modelling of probabilistic occupant behaviour in buildings with a physical multi-domain impact on both thermal and electrical aspects. The behaviour considering occupancy [20], the use of lighting [22] and the use of use of appliances [17] in dwellings has been implemented, but the same method can be used for other stochastic behaviour (e.g. opening of windows by occupants). The implemented stocastic model is largely consistent with the model of Richardson et al. [21]. The output of the model are presence and activity profiles of the building occupants, coupled to the usage of electric appliances and lighting which result in (i) convective $Q_{c}$ and radiative $Q_{r}$ internal heat gains and (ii) the real or active electric power demand $P$. The model is integrated in the dynamic simulation of the thermal response of buildings and its grid impact towards the assessment of distributed generation at district scale.

Many researchers [10, 18, 19, 20, 21, 22, 23, 25, 30] use Markov properties for modelling occupancy and the use of appliances in buildings. A Markov process is a stochastic process $\left\{X_{t}, t \geq 0\right\}$ with values in a state space $E$ where for any $s<t$ and any measurable set $A \subset E$ holds that $P\left(X_{t} \in A \mid X_{r}, 0 \leq r \leq s\right)=$ $P\left(X_{t} \in A \mid X_{s}\right)=P\left(s, X_{s}, t, A\right)$ where the function $P\left(s, X_{s}, t, A\right)$ describes the probability that the process is in $A$ at $t$ conditioned by the information that the process is in $x$ at time $s$ [15]. The Markov property depicts that the future state does not depend on how the current state is obtained but only depends on the present state itself. The process is characterized by the transition probabilities $A \rightarrow P\left(s, X_{s}, t, A\right)$ parameterized by $s, x$ and $t$.

Within this work, both embedded discrete time Markov chains and semi-Markov processes are implemented.

\subsection{Occupancy: Embedded discrete time Markov chains}

Occupancy in buildings is typically dealed with as embedded discrete time Markov chains [18, 20, 23, 30] however also semi-Markov processes may be found suitable [19], i.e. especially for single-person offices where only two states are possible. Allthough used only for evaluation of uccpancy, the implemented model for embedded discrete time Markov chains is generic and can be used and extended for all similar Markov chains.

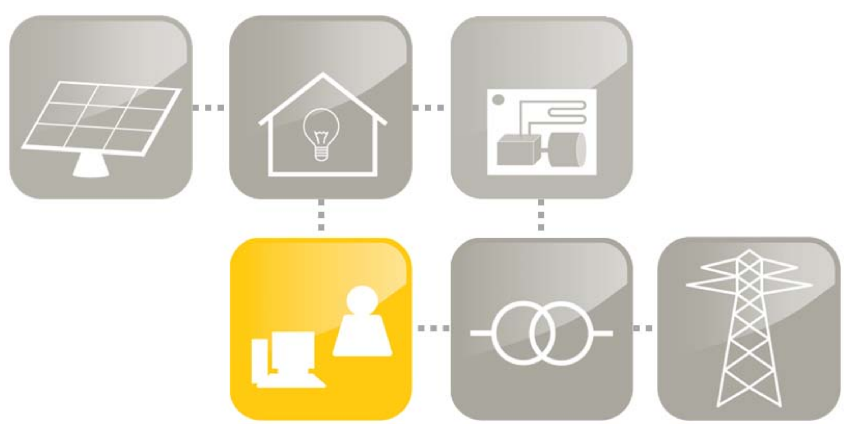

Figure 1: Occupant behaviour as part of the simulation of energy networks at district scale by the K.U.Leuven Energy Institute.

In an embedded discrete time Markov Chain, the possible change in occupancy (i.e. a person entering or leaving the building) is evaluated repeatingly after a discrete time step (i.e. mostly 10 minutes) based on the previously descried Markov property $P\left(s, X_{s}, t, A\right)$. The Markov-properties are stored in records as record Occupance(Integer $n(\min =1)$,SI.Time period,

SI.Time $s$, Real[ $\left.[s, n+1, n+1] T_{w d}, T_{w e}\right)$;

where $n$ depicts the number of different states, period the total time span across which the Markov process transition probabilities repeat themselves (i.e. mostly 24 hours) and $s$ the number of equal time steps within the period wherefor the transition probabilities are given.

The matrices $T_{w d}$ and $T_{w e}$ depict the transition probabilities of the Markov process for a work day and weekend respectively so that $P\left(s, X_{s}, t, A\right)=$ $T\left[s, X_{s}+1, A+1\right] . T_{w d}$ and $T_{w e}$ are retrieved from Richardson et al. [20] where they are given for dwellings with 1 to 5 inhabitants and where the possibility of correlatioin between differen inhabitants for arrival or departure is included. At each discrete time step, the the actual current (cumulated) transition probabilities based on the knowledge of the present state and the time of day are extracted from the $\mathbf{R}^{3}$-matrix containing all transition probabilities and evaluated for possible state changes.

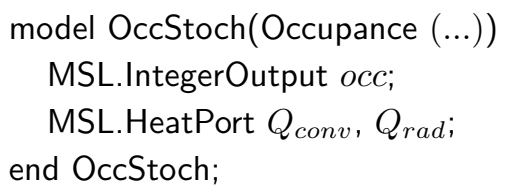

The presence of inhabitants determines internal gains from people and the possibility of opening windows, whereas it is a necessary condition for the use of many electric appliances and the use of lighting in buildings. 


\subsection{Appliances: Semi-Markov processes}

Differently from the evaluation of occupancy in buildings, the use of appliances is generally implemented as semi-Markov processes [19, 21, 22, 25, 30].

For the use of many domestic electric appliances, presence of at least one of the occupants is a first necessary condition, derived from the previously mentioned embedded discrete time Markov chains.

Secondly, the activity profile of humans depends on the moment of the day. Activity profiles are generally described as a sequence of probabilities denoting the chance that a certain activity occurs at the time of a day. The probabilities are depicted independently from the current state (i.e. doing the activity or not) but depending on the number of active occpants, differently from the description of human occupancy in buildings by transition probabilities which depend on the current state.

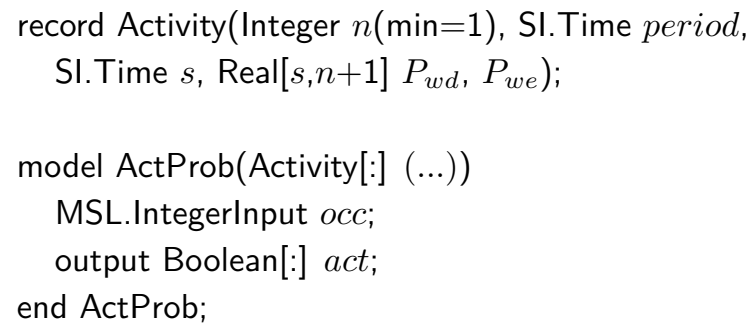

where $n$ depicts the number of occupants active at moment $t$ derived from the stochastic occupancy profile for which the stated probabilities count, period the total time span across which the Markov process probabilities repeat themselves (i.e. mostly 24 hours) and $s$ the number of equal time steps within the period wherefor the transition probabilities are given. The matrices $P_{w d}$ and $P_{w e}$ depict the probabilities of the Markov process for a work day and weekend respectively. The different probabilities are derived from Richardson et al. [21] for the activities watching television, cooking, doing laundry, ironing, cleaning the house and spending time on washing and clothing. The library of activites can be extended with additional data from time-consumption surveys [28]. Currently, the implementation only takes into account the different profiles for working days and weekend days, but can be easily expanded e.g. for taking into account long days of absence or vacation.

At each discrete time step, the activity probability is evaluated for possible use of appliances related to the activity based on the relative use of appliances. When the decission is taken that a certain appliance is switched on, the length during witch the appliance will remain on is determined once, differently from the occupancy pattern where the possible switch is evaluated for every discrete time step. This simplification of an embedded discrete time Markov chain into a semiMarkov process is only possible if only two states occur, i.e. off and on in this case, resulting in less simulation events and thus shorter simulation times. The drawback of this approach is that interactions from one occurance to another are excluded, i.e. it excludes adaptation and intermediate states.

The data implementation happens as
record Appliance(Activity act, Real $n_{\text {cycle }}$, Real cal,
Real $f_{\text {rad }}$, Real $f_{c o n v}$, SI.Time $l_{c y c l e}$, SI.Power $P_{c y c l e}$, SI.Power $P_{\text {standby }}$ );

record Light(Integer $n$,SI.Power[n] $\left.P_{\text {cycle }}\right)$;

where act is the required activity of the appliance, $n_{\text {cycle }}$ is the average number of cycles during a year, cal a calibration scalar defining the relation between the activity and the effective use of the appliance, and $l_{c y c l e}$ is the average length of usage, where $P_{\text {cycle }}$ and $P_{\text {standby }}$ are the total power demand of the appliance when switched on and at standby modus respectively and where $f_{\text {conv }}$ and $f_{\text {rad }}$ are the convective and radiative fraction respectively of the local heat production by the appliances.

For determination of the duratioin for which an appliance remains on, the distribution of the duration $P(t)$ for the use of lighting is fitted as $0.1664 \ln (t)+$ 0.1084 with a $r^{2}$ value of 0.9961 [21] and where $t$ is the duration in minutes. The duration probability for the use of other appliances is set equal to the average usage with a standard deviation of 10 percent except for the television where a $P(t)$ of $1+1.021 \exp \left(t^{0.91}\right)$ is given [21].

\subsection{Physical impact}

As mentioned earlier, human behaviour in buildings has a multi-physical impact. Occupants presence and the use of electric appliances determine the internal heat gains in a building, whereas the electric appliances (together with possible local electric generation, e.g. by means of a photovolaic system) determine the load of a building on the electric distribution grid affecting the grid voltage.

Both the occupancy of humans in the building and the use of electric appliance result in internal gains in a building, influencing both thermal comfort and the related energy consumption for heating (and cooling). 
For both human presence and each different implemented appliance, the internal heat production is depicted as a (long-wave) radiative $f_{\text {rad }}(-)$ and convective $f_{\text {conv }}(-)$ fraction. So far, dynamic effects such as wasted heat (e.g. for cooking or washing) or temperature-dependent fractions depending on the cycle are not included.

The fractions of $f_{\text {rad }}$ and $f_{c o n v}$ for different appliances and humans are derived from ASHRAE Fundamentals [2]. When no value is available, a division of $f_{\text {rad }}=f_{\text {conv }}=0.5$ is set.

On the electric side, all loads of the the used appliances are seen as active loads $P(W)$. Differently from e.g. heat pumps or motors, where also the reactive power $S$ is of importance.

\subsection{Random number generation}

For the purpose of probabilistic simulation, the 4-cycle generation algorithm for pseudo-random numbers of Wichmann and Hill [29] is implemented combining a long period of $2^{121}$ with a small size of state of 16 bytes. Taking into account a discrete time step of 1 minute and the number of stochastic processes in a classic dwelling (i.e. in the order of size between 30 and 100 depending on the number of occupants and appliances) regarding building occupant behaviour, the period of the random generator remains in the order of size of $2^{90}$ making it suitable for the mentioned modelling purpose as no repitition will occur during a simulation.

\section{Physical relevance}

The stochastic behaviour of building occupants influences both the electric consumption of the used domestic appliances and the heat production of the same domestic appliances and the occupants. The results show both similiarities (i.e. similar averages) as large differences (i.e. large deviations) from the deterministic approach as in ISO 13790 [12] where a deterministic scheme for simulation purposes is depicted on the internal gains from occupants and appliances (see Fig. 2,3,4). The standard differentiates three different time zones between 7 pm, 17 am and 23 for the internal gains.

In order to quantify the difference between a stochastic and deterministic approach, a single-zone reference building is modelled (see appendix A) and 5 identical models (with each the same deterministic or different stochastic profile respectively) are coupled to
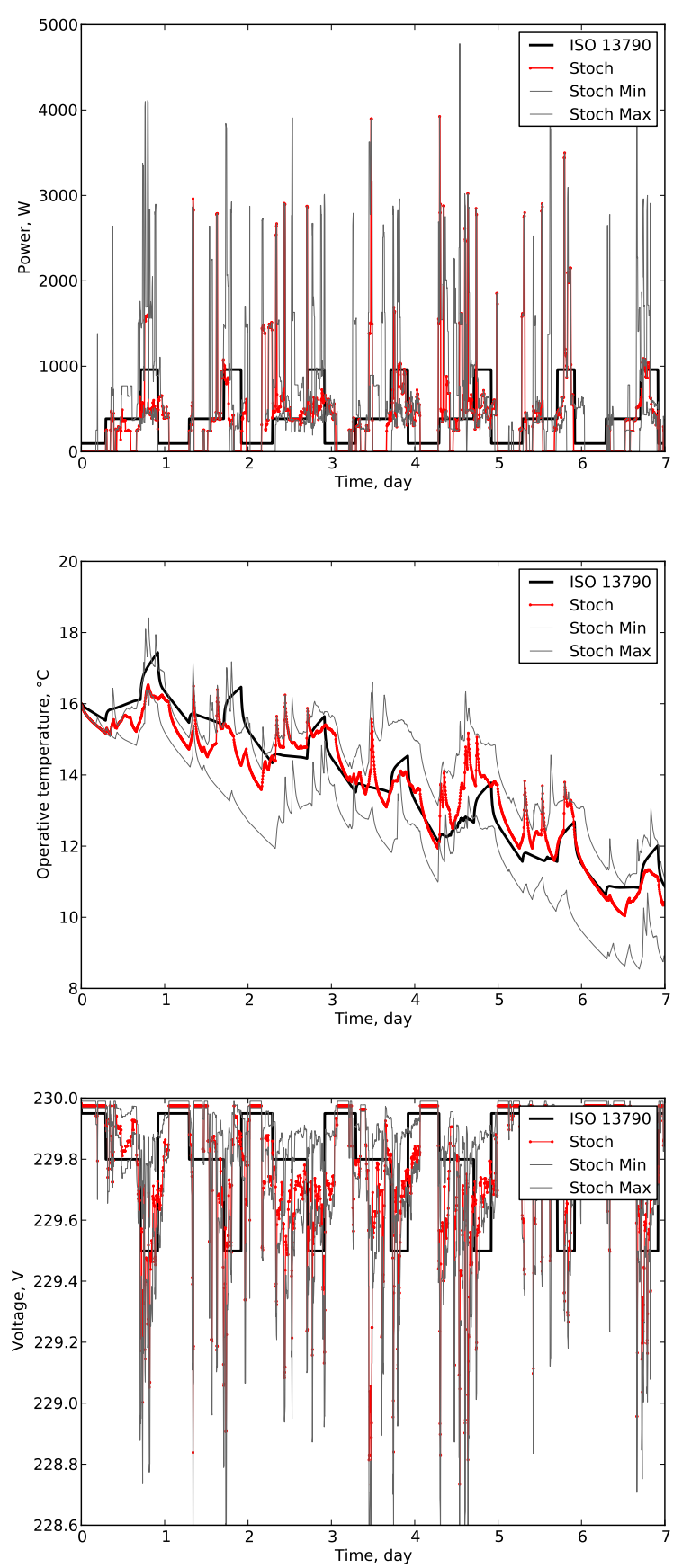

Figure 2: Week profile of (a) the total power, (b) the indoor operative temperatures of a building in free run and (c) the resulting voltage in an example grid as deterministically described in (ISO 13790) and (Stoch) with a stochastic profile of occupancy, lighting and electric appliances. The minima (Stoch min) and maxima (Stoch max) of the results for 1 tot 5 inhabitants are given for the same situation with the same appliances.

an electric grid (see appendix B). For quantification of the second-order effects of heating, also an ideal heating system has been introduced (see appendix A). 

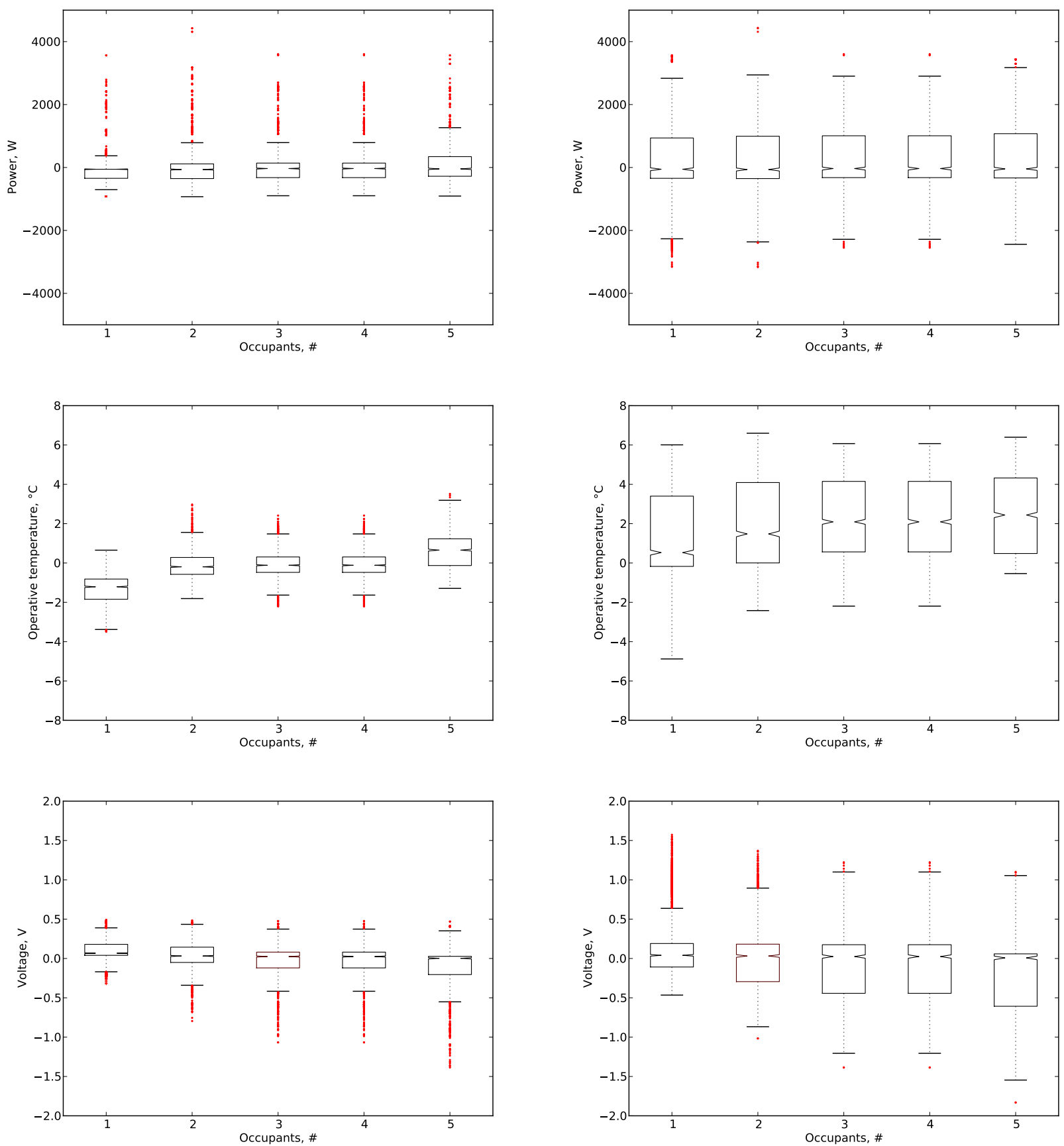

Figure 3: Standard deviations $(\sigma,-\sigma)$ representend by the boxes, $(3 \sigma,-3 \sigma)$ representend by the bars, and tails of difference between the stochastic and determinstic approach for (a) the total electric power demand, (b) the indoor operative temperatures of a building in free run and (c) the resulting voltage in an example grid of the difference between a stochastic profile of occupancy, lighting and electric appliances and as described in ISO 13790 for 1 tot 5 inhabitants.

\subsection{Thermal load}

The profile of total stochastic and deterministic thermal power (see Fig.2a) results in convective and radia-

Figure 4: Standard deviations $(\sigma,-\sigma)$ representend by the boxes, $(3 \sigma,-3 \sigma)$ representend by the bars, and tails of difference between the stochastic and determinstic approach for (a) the total electric power demand, (b) the indoor operative temperatures and (c) the resulting voltage in an example grid of the difference between a stochastic profile of occupancy, lighting and electric appliances and as described in ISO 13790 for 1 tot 5 inhabitants and a building including ideal heating. 
tive internal gains for the building and determines the internal temperature (see Fig.2.b), the required thermal energy for thermal comfort and the possibility of overheating.

Comparison between the stochastic and deterministic profile of total power (see Fig.2a,3a) shows both similarities as differences: The difference between the determinsitic and stochastic load profiles averages nearly zero determining that the total load is similar, whereas also the standard deviation $\sigma$ is very small in the order of size of $0.3 \mathrm{~kW}$ (see Fig.3a) denoting that also the overall or average day profile, i.e. the moment of the day internal loads occur, is similar in both the deterministic and stochastic profile. In contrast, high peaks e.g. resulting from cooking occur in the stochastic profile which disappear in the deterministic approach (resulting in the long tail in Fig.3a).

As the average internal loads are alike between the deterministic and stochastic approach, also the thermal response of an example dwelling (see appendix A) shows small deviations (see Fig.2b,3b) with a standard deviation $\sigma$ in the order of size of 0.5 Kelvin. As the thermal building response of a dwelling is slow due to a relatively high thermal mass, the effect of the high peaks in the stochastic internal gains remains small compared to a deterministic approach.

\subsection{Electric load}

The profile of total stochastic and deterministic electric power results in electric currents in the distribution grid and determines the voltage drops (see Fig.2c).

Similar to the thermal load, as the average electric loads are alike between the deterministic and stochastic approach (see Fig.2a), also the average electric response of an example grid (see appendix B): the voltage drop shows small deviations (see Fig.3c) with a standard deviation $\sigma$ in the order of size of 0.2 Volt.

In contrast to the conclusion on peak loads on the thermal side, electric peak loads have a direct influence on the voltage of an electric grid which disappears in the deterministic approach (see Fig.2c,3c). Even more, the effect of peak loads accumulates at grid-level: as (i) in real life and in the stochastic approach of occupant behaviour not all peaks occur at the same time and (ii) a peak load not only influences the observed voltage at the respective dwelling but also the the observed voltage at the adjacent dwellings between the respective dwelling and the grid source, more and higher voltage drops are noticed in the stochastic approach shown by the long tail of 0.5 to 1.0 Volt in Fig.3c.

As the voltage in a low-voltage distribution grid is not allowed to drop below $207 \mathrm{~V}$ and the strongest voltage drops are caused by peak loads, the use of deterministic profiles may strongly underestimates the possible problem of grid-instability.

\subsection{Coupled thermal-electric load}

Both the influence on indoor temperature and voltagedrops by the stochastic modelled internal gains are first-order effects as they are directly influenced by the bottom-up modelled power profile. As yet described in the introduction, new and future a (zero-energy) buildings results more often in an all-electrical solution with a combination of building-integrated photovoltaic systems (BIPVs) and an electrical heat pump, a trend yet noticeable in existing low-energy dwellings. As the stochastic profile of both occupancy and internal heat gains determine both the switch-on and -off conditions for the electric heating - and thus also the electric peak loads of the heat pump towards the grid - also a secondorder effect can be noticed. In order to visualize this effect, an ideal heating system is implemented in the example dwelling (see appendix A).

\subsubsection{Thermal origin}

Comparison between the stochastic and deterministic profile of total power demand (see Fig.4a) shows both similarities as differences: The difference between the determinsitic and stochastic load profiles averages nearly zero determining that the total load for both the appliances ánd heating is similar. Differently from the original total load in Fig.3a, the standard deviation $\sigma^{+}$ and $3 \sigma$ overall become high (see Fig.4a) in the order of 1 and $2 \mathrm{~kW}$ respectively. The high deviations $\sigma^{+}$ and $3 \sigma$ denote that - allthough the total energy demand remains similar- the time shift and the number of peaks in heat load between a deterministic and stochastic profile is signficant

Due to shorter intervals without heating in the stochastic profile compared to the deterministic, a 1 to $2 \mathrm{~K}$ higher average operative indoor temperature is noticed in the stochastic approach (see Fig.4b). The high $\sigma$ and $3 \sigma$ are due to comparison between non-heated and heated moments as the moments of heating are not the same in the different situations.

\subsubsection{Electric effect}

Similar to results concerning the thermal load, as the average electric loads are alike between the deterministic and stochastic approach (see Fig.4a), also the aver- 
age electric response of an example grid (see appendix B) by means of a voltage drop shows small deviations (see Fig.4c).

Allthough the average response remains the same, deviations $\sigma$ and $3 \sigma$ of up to 0.5 and $1.5 \mathrm{~V}$ repspectively are found, (see Fig.4c) with a long tail in the opposite direction as may be noticed in Fig.3c. As mentioned earlier, the effect of peak loads accumulates at grid-level. In real life and in the stochastic approach of occupant behaviour not all peaks occur at the same time and a peak load not only influences the observed voltage at the respective dwelling but also the the observed voltage at the adjacent dwellings between the respective dwelling and the grid source, resulting in high $\sigma$ and $3 \sigma$. The long tail in the opposite direction as Fig. $3 \mathrm{c}$ is caused by the deterministic profile where - in condtradiction to the stochastic profile - all dwellings require heat at exactly the same moment resulting in strong grid loads.

\section{Conclusions}

An integrated approach of probabilistic occupant behaviour in buildings with a physical multi-domain impact has been modelled and presented. The human behaviour considering occupancy, the use of lighting and the use of electric appliances in dwellings has been implemented and is used for simulation of coupled thermal and electrical systems in the building stock. Implementing stochastic occupant behaviour influences the internal heat gains which in turn influences the heat load of the building and the switch-on and -off moment of e.g. an electric heat pump. This, together with the power demand of the used electric appliances and possible on-site generation determine the load on the electric grid and possible instabilities.

By means comparison for a reference building zone and example grid, the importance of stochastic modelling of occupant behaviour in dwellings at both the building and district scale. Comparison between a determinsitic approach as proposed in ISO 13790 and the use stochastic profiles shows that the direct first order effect is on average rather small: the difference in total internal gains and its influence on the indoor temperature averages nearly zero and the standard deviations $\sigma$ are small, however high peaks may occur. Also the difference in effect on the electric distribution grid voltage averages nearly zero, however here strong peaks occur which are of most importance for the grid stability. When taking in account the second order effect of heating by means of electricity, much larger differ- ences are noticed: due to longer and more differentiated occupancy times, the average indoor temperature rises. Furthermore, the moment of heating differentiates compared to a determinsitic approach resulting in more but smaller peak demands towards the electricity grid.

\section{Acknowledgements}

The authors gratefully acknowledge the K.U.Leuven Energy Institute (EI) for funding this research through granting the project entitled Optimized energy networks for buildings.

\section{A Building model}

A high-order lumped capacitance model for predicting the unsteady building response is developed within Modelica [5, 27]. Solar radiation absorbed by the exterior surface is implemented based on the incident solar irradiation as found by the calculations in the External package depending on time, inclination and orientation $\mathrm{s}$ and the short-wave absorption coefficient of the surface. Long wave radiation between the surface and environment is determined based on the retrieved sky temperature. The convective gains and the resulting change in air temperature of a thermal zone are modelled as a thermal circuit based on convective heat exchange with the walls and ventilation. Similar to the model for a wall, a thermal circuit formulation for the direct radiant exchange between surfaces can be derived. The heat exchange by long-wave radiation is simplified by means of a delta-star transformation [13] and definition of a radiant star node. The diffuse solar gains are divided based on the surface and emissivity of all surfaces. For direct solar gains, a factor for each surface can be given as parameter of the zone, to which direct solar gains will be divided. The star node formulation of both convective and radiative heat exchange in a thermal zone allows a straight-forward formulation for the influence of internal gains by adding them in the heat balance of radiant star node and air node of the zone of interest. Comparative literature [16] shows that the made simplifications remain a high accuracy.

The thermal model of a window is similar to the model of an exterior wall but includes the absorption of solar irradiation by the different glass panes and the transmission to the adjacent indoor zone. The properties for absorption by and transmission through the glazing are taken into account depending on the angle of incidence of solar irradiation and are based on the 
output of the WINDOW 4.0 software [8] as validated by Arasteh [1] and Furler [9]. The transmitted diffuse short-wave solar radiation is treated to strike all room surfaces weighted to their surface and emissivity, whereas the direct short-wave solar gains are modeled to fall mainly on the floor. However, only small differences would arise when making different assumptions on the distribution of the transmitted energy [16].

A meteo model is implemented in the simulation environment and data are derived from Meteonorm 6.1 for Uccle, Belgium. The zenith angle of an inclined surface is calculated internally [11] whereby the anisotropic sky dome model presented by Skartveit and Olseth $[24,6]$ is implemented.

The ideal heating system consists of an unlimited convective power controlled by a set point of 21 degrees Celcius for the operative temperature when occupancy occurs.

The reference building zone measures $36 \mathrm{~m}^{2}$ by 3 $m$ representing the dayzone of an average house. The construction consists of a highly-insulated cavity wall with $20 \mathrm{~cm}$ of mineral wool and a large south-facing double-pane window. For the stochastic determination of user behaviour, 1 to 5 occupans have been implemented respectively, an array of 16 bulbs has been taken into account and 10 electric appliances (i.e. a hob, a micro wave, two TV's, a vacuum cleaner, a hifi, an iron, a pc, a dishwasher and a washingmachine) are modelled.

\section{B Grid model}

The example grid for modelling grid voltage drops used within this work consists of a simplified grid based on the Modelica Standard Library 3.1, consisting of a constant voltage source of $230 \mathrm{~V}$ and lossy RC-lines. The RC-lines have each a capacitance of 0.1 $m F / m$, a resistance of $0.05 \mathrm{~m} \Omega / \mathrm{m}$ and have a length of $100 \mathrm{~m}$. Here, 5 dwellings are coupled in parallel to the grid. The power demand of the dwelling determines the electric current influencing the grid voltage at both the dwelling and the neighbouring dwellings.

Note that the implemented model only serves to interpret the importance and impact of stochastich modelling of occupancy behaviour in coupled thermal and electric systems in buildings. Within the same project of the K.U.Leuven Energy Institute mentioned in the acknowledgements, a detailed grid model is developed.

\section{References}

[1] D K Arasteh, J Hartmann, and M Rubin. Experimental verification of a model of heat transfer through windows. ASHRAE Transactions, 93(1):1425-1431, 1986.

[2] ASHRAE. 2009 ASHRAE Handbook: fundamentals. ASHRAE American Society of Heating Refrigerating and Air-Conditioning Engineers, Atlanta, 2009.

[3] R Baetens, R De Coninck, L Helsen, and D Saelens. The impact of domestic load profiles on the potential of building integrated photovoltaic systems in extremely low-energy dwellings. In Renewable Energy Research Conference, pages 3-14, Trondheim, June 7-8, 2010.

[4] R Baetens, R De Coninck, L Helsen, and D Saelens. The impact of the heat emission system on the grid-interaction of building integrated photovoltaics in low-energy dwellings. In 8th International Conference on System Simulation in Buildings, page P137, 2010.

[5] J Clarke. Energy simulation in building design. ButterworthHeinemann, Oxford, 2nd ed. edition, 2001.

[6] S Darula, R Kittler, and C Gueymard. Reference luminous solar constant and solar luminance for illuminance calculations. Solar Energy, 79(5):559-565, November 2005.

[7] Roel De Coninck, Ruben Baetens, Bart Verbruggen, Dirk Saelens, Johan Driesen, and Lieve Helsen. Modelling and simulation of a grid connected photovoltaic heat pump system with thermal energy storage using Modelica. In Philippe Andre, Stephane Bertagnolio, and Vincent Lemort, editors, proceedings of the 8th International Conference on System Simulation in Buildings, Liege, 2010.

[8] E U Finlayson, D K Arasteh, C Huizenga, M D Rubin, and M S Reilly. WINDOW 4.0: Documentation of calculation procedures, 1993.

[9] R A Furler, P Williams, and F K Kneubühl. Experimental and theoretical studies on the energy balance of windows NEFF Project report 177.1, 1988.

[10] P Hoes, J Hensen, M Loomans, B Devries, and D Bourgeois. User behavior in whole building simulation. Energy and Buildings, 41(3):295-302, March 2009.

[11] M Iqbal. An introduction to solar radiation. Academic Press Inc, New York - London, 1983.

[12] ISO/FDIS 13790. Energy performance of buildings - Calculation of energy use for space heating and cooling, volume 2007. 2008.

[13] A E Kenelly. Equivalence of triangles and stars in conducting networks. Electrical World and Engineer, 34:413-414, 1899.

[14] S A Klein. TRNSYS - A transient simulation program, 1973.

[15] A N Kolmogorov. Uber die analytischen Methoden in der Wahrscheinlichkeitsrechnung. Mathematische Annalen, 104:415-458, 1931.

[16] R J Liesen and C O Pedersen. An evaluation of inside surface heat balance models for cooling load calculations. ASHRAE Transactions, 103 Part 2:485-502, 1997.

[17] J V Paatero and P D Lund. A model for generating household electricity load profiles. International Journal of Energy Research, 30(5):273-290, April 2006.

[18] J Page, D Robinson, N Morel, and J Scartezzini. A generalised stochastic model for the simulation of occupant presence. Energy and Buildings, 40(2):83-98, 2008. 
[19] W Parys, D Saelens, and H Hens. Coupling of dynamic building simulation with stochastic modelling of occupant behavior in offices - a review-based integrated methodology. Journal of Building Performance Simulation, Accepted, 2010.

[20] I Richardson, M Thomson, and D Infield. A high-resolution domestic building occupancy model for energy demand simulations. Energy and Buildings, 40(8):1560-1566, 2008.

[21] I Richardson, M Thomson, D Infield, and C Clifford. Domestic electricity use: A high-resolution energy demand model. Energy and Buildings, 42(10):1878-1887, October 2010.

[22] I Richardson, M Thomson, D Infield, and A Delahunty. Domestic lighting: A high-resolution energy demand model. Energy and Buildings, 41(7):781-789, 2009.

[23] D Robinson, N Campbell, W Gaiser, K Kabel, A Lemouel, N Morel, J Page, S Stankovic, and A Stone. SUNtool - A new modelling paradigm for simulating and optimising urban sustainability. Solar Energy, 81(9):1196-1211, September 2007.

[24] A Skartveit and J A Olseth. Modelling slope irradiance at high latitudes. Solar Energy, 36:526-541, 1986.

[25] M Stokes, M Rylatt, and K Lomas. A simple model of domestic lighting demand. Energy and Buildings, 36(2):103-116, 2004.

[26] P Strachan, G Kokogiannakis, and I Macdonald. History and development of validation with the ESP-r simulation program. Building and Environment, 43(4):601-609, April 2008.

[27] C P Underwood and F W H Yik. Modelling methods for energy in buildings. Blackwell Publishing, 2004.

[28] D Vanneste, P De Decker, and I Laureyssen. Woning en woonomgeving in België, 2001.

[29] B A Wichmann and I D Hilll. Generating good pseudorandom numbers. Computational Statistics \& Data Analysis, 51(3):1614-1622, 2006.

[30] J Widén and E Wäckelgå rd. A high-resolution stochastic model of domestic activity patterns and electricity demand. Applied Energy, 2009. 